\title{
SOBRE UMA PEQUENA COLEÇÃO DE ÁCAROS (ARTHROPODA, ACARI) DO TERRITÓRIO FEDERAL DE FERNANDO DE NORONHA, BRASIL. II. ORIBATÍDEOS ${ }^{1}$
}

Carlos H.W. Flechtmann ${ }^{2}$

RESUMO: O exame de uma coleta feita em setembro de 1987 na Ilha de Fernando de Noronha, revelou a presença de duas espécies de ácaros oribatideos filiados à familia Phtiracaridae, a saber: Atropacarus (Hoplophorella) scapellus Aoki, 1965 e A. (H.) Schauenbergi Mahunka, 1978, em matéria orgânica acumulada sobre o ramo de uma árvore, a 1,8 $\mathrm{m}$ de altura sobre o solo. Termos para Indexação: ácaros, oribatídeos, Fernando de Noronha.

\section{ON A SMALL COLLECTION OF MITES (ARTHROPODA, ACARI) FROM THE ISLAND OF FERNANDO DE NORONHA, BRAZIL. II. ORIBATEI}

ABSTRACT: Two species of Phthiracaridae oribatid mites, namely Atropacarus (Hoplophorella) scapellatus Aoki, 1965 and $A$. (H.) schauenbergi Mahunka, 1978, are reported from debris accumulated on a tree branch about $1.8 \mathrm{~m}$ above soil surface, in Fernando de Noronha Island, Brazil.

Index Terms: mites, oribatei, Fernando de Noronha.

1 Com auxílio do Conselho Nacional de Desenvolvimento Cientrfico e Tecnológico - CNPq

2 Departamento de Zoologia, USP / ESALQ, 13400 - Piracicaba - SP. 


\section{INTRODUÇÃo}

Na literatura sobre ácaros no Território Federal de Fernando de Noronha é assinalada a presença de várias espécies da poeira domiciliar, todas pertencentes ao grupo Astigmata (GALVÃo \& GUITTON, 1986) e de ácaros de plantas do grupo Prostigmata, de um carrapato, grupo Metastigmata e de uma espécie de Astigmata (FLECHTMANN, 1987). Não foram encontradas referências a ácaros oribatídeos (grupo Cryptostigmata).

\section{MATERIAL E MÉTODOS}

No período de 07 a 10 de setembro de 1987, cerca de 3 meses após a última chuva, foram coletadas amostras de matéria orgânica (detritos) acumulada nas axilas dos ramos de árvores. De uma destas amostras coletada a $1,8 \mathrm{~m}$ acima do nivel do solo, foram recuperados 43 espécimes de ácaros.

\section{RESULTADO E DISCUSSÃO}

Os 43 espécimes obtidos pertencem ao grupo de ácaros oribatídeos de prossoma pticóide, ou seja, que são capazes de dobrar o prossoma de encontro ao histerossoma, e incluidos na familia Phthiracaridae.

Estes ácaros foram enviados ao Dr. W. Niedbala, na Polônia, que identificou 41 individuos como pertencentes à espécie Atropacarus (Hoplophorella) scapellatus Aoki, 1965 e 2 individuos como pertencentes à espécie $A$. (H.) schauenbergi Mahunka, 1978.

E este o primeiro relato da ocorrência destas 2 espécies na região Neotropical. Ácaros do gênero Atropacarus, porém, não identificados especificamente, já foram coletados 
de folhas em decomposição sobre o solo de sitios florestais da Amazônia Central (RIBEIRO, 1986).

\section{REFERÊNCIAS BIBLIOGRÁFICAS}

FLECHTMANN, C.H.W. Sobre uma pequena coleção de ácaros (Arthropoda, Acari) do Território Federal de Fernando de Noronha,Brasil. Anais da ESALQ, Piracicaba-SP, 44: 1643$1647,1987$.

GALVÃo, A.B. \& N. GUITTON. Ácaros em poeira domiciliar das capitais brasileiras e Ilha de Fernando de Noronha. Memórias do Instituto Oswaldo Cruz, Rio de Janeiro, 81 (4): 417-430, 1986 .

RIBEIRO, E.F. Oribatídeos (Acari, oribatida colonizadores de folhas em decomposição sobre o solo de três sitios florestais da Amazônia Central. Manaus, 1986. 178p. (Mestrado, Instituto Nacional de Pesquisas da Amazônia).

Trabalho entregue para publicação em 02.01 .91 Trabalho aprovado para publicação em 06.06.91 